\title{
A Case of Poorly Differentiated Squamous Cell Carcinoma in the Breast: A Thorough Investigation of its Origins
}

\author{
Fumie Shono ${ }^{1,4}$, Shigeki Inui ${ }^{*}, 4$, Satoshi Takagi ${ }^{1}$, Kazuya Kuraoka ${ }^{2}$, Kiyomi Taniyama ${ }^{2}$, \\ Shinichi Ansai $^{3}$ and Tetsunori Kimura ${ }^{3}$
}

\author{
${ }^{I}$ Department of Plastic and Reconstructive Surgery and ${ }^{2}$ Institute for Clinical Research, National Hospital Organization \\ Kure Medical Center and Chugoku Cancer Center, Hiroshima, Japan \\ ${ }^{3}$ Sapporo Institute for Dermatopathology, Hokkaido and ${ }^{4}$ Department of Regenerative Dermatology, Graduate School of \\ Medicine, Osaka University, Osaka, Japan
}

\begin{abstract}
An 81-year-old female consulted us because of an ulcerated tumor in her left breast of several years' duration. Partial biopsy showed that the tumor consisted of anaplastic squamous cells, indicating squamous cell carcinoma from unknown origin. CT images demonstrated tumors in the left breast, liver and bile duct. However, further investigation was not possible due to the patient's refusal. To avoid possible localized complication caused by tumor rupture and/or infection, the breast tumor was totally excised. Histopathology revealed that irregularly shaped tumor nests had invaded the entire dermis resulting in massive necrosis. The tumor cells were of various sizes and shapes, and showed individual keratinization and atypical mitotic figures. In addition, other larger masses consisted of hyperchromatic small squamous cells with basophilic cytoplasms. These cells were poorly differentiated without apparent keratinization. Immunohistochemically, the excised tumor was stained strongly positive for epidermal growth factor receptor, partially positive for epithelial membrane antigen and BerEP4 but negative for cytokeratins 5, 6 and 20, estrogen receptor, progesterone receptor, ErbB2/HER2, gross cystic disease fluid protein-15, carcinoembryonic antigen and S-100. Since these findings were not sufficient to identify the tumor origin, we carefully rechecked the histopathology of the surgical specimens and found irregular elongation at the periphery of the overlying epidermis, which contained atypical keratinocytes with pleomorphic nuclei, suggesting a diagnosis of Bowen's disease. We therefore concluded that tumor cells of Bowen's disease in the epidermis, independently from the tumors of liver and bile duct, had invaded downward, finally reaching the breast tissue.
\end{abstract}

Keywords: Squamous cell carcinoma, Bowen's disease, SCC in situ, basal-like subtype of breast cancer.

\section{CASE PRESENTATION}

An 81-year-old female visited us because of an ulcerated tumor in her left breast of several years' duration (Fig. 1A, arrows). Erythematous plaques adjacent to the tumor were seen (Fig. 1A, triangles). Since the patient had injured herself by accidentally falling down from her bed onto the floor, both purpura and erosions, unlikely to have been caused by the tumor, were observed on the breast. Axillary lymph nodes were not palpable. A partial biopsy showed that the tumor consisted of anaplastic squamous cells, indicating squamous cell carcinoma (SCC) from unknown origin. Laboratory findings showed that complete blood cell count and biochemical values were within normal limits. However, $\alpha$-fetpoprotein (AFP) in the blood was moderately elevated to $67.2 \mathrm{ng} / \mathrm{ml}$ (normal: $<10 \mathrm{ng} / \mathrm{ml}$ ) and CA19-9 markedly increased to $497 \mathrm{U} / \mathrm{ml}$ (normal: $<37 \mathrm{U} / \mathrm{ml}$ ) while other tumor markers such as SCC, CA125 and PIVKA-II were normal. CT images demonstrated tumors in the left breast, liver and bile duct. However, because the patient and her family refused biopsy and treatment for the latter two tumors, we

*Address correspondence to this author at the Department of Regenerative Dermatology, Graduate School of Medicine, Osaka University, 2-2 G2, Yamada-oka, Suita-shi, Osaka 5650871, Japan; Tel: +81-6-6879-3960; Fax: +81-6-6879-3962; E-mail: inui@r-derma.med.osaka-u.ac.jp could not conduct further investigations. them On the other hand, informed consent was obtained from the patient and her family for total excision of the breast tumor under general anesthesia to avoid possible localized complication caused, for example, by tumor rupture or infection. Gross findings showed that the resected tissue block measured $17 \times 8 \times 3 \mathrm{~cm}$ and that the tumor was $25 \times 17 \times 22 \mathrm{~mm}$ in size and had invaded the breast from the nipple to the mammary gland. Simultaneously performed sentinel lymph node biopsy using indigo carmine and one of three stained lymph nodes was positive for metastasis. Histopathology revealed that irregularly shaped tumor nests had invaded the entire dermis (Fig. 1B), resulting in massive necrosis (Fig. 1C). The tumor cells were of various sizes and shapes, and showed individual keratinization and atypical mitotic figures (Fig. 1D). In addition, the other comparatively larger masses consisted of hyperchromatic small squamous cells with basophilic cytoplasms (Fig. 1E). These cells were poorly differentiated without apparent keratinization. Immunohistochemically, the excised tumor was stained strongly positive for epidermal growth factor receptor (EGFR) (Fig. 1F), partially positive for epithelial membrane antigen (EMA) and BerEP4 but negative for cytokeratins 5, 6 and 20, estrogen receptor (ER), progesterone receptor (PR), ErbB2/HER2, gross cystic disease fluid protein-15 (GCDFP$15)$, carcinoembryonic antigen (CEA) and S-100. 


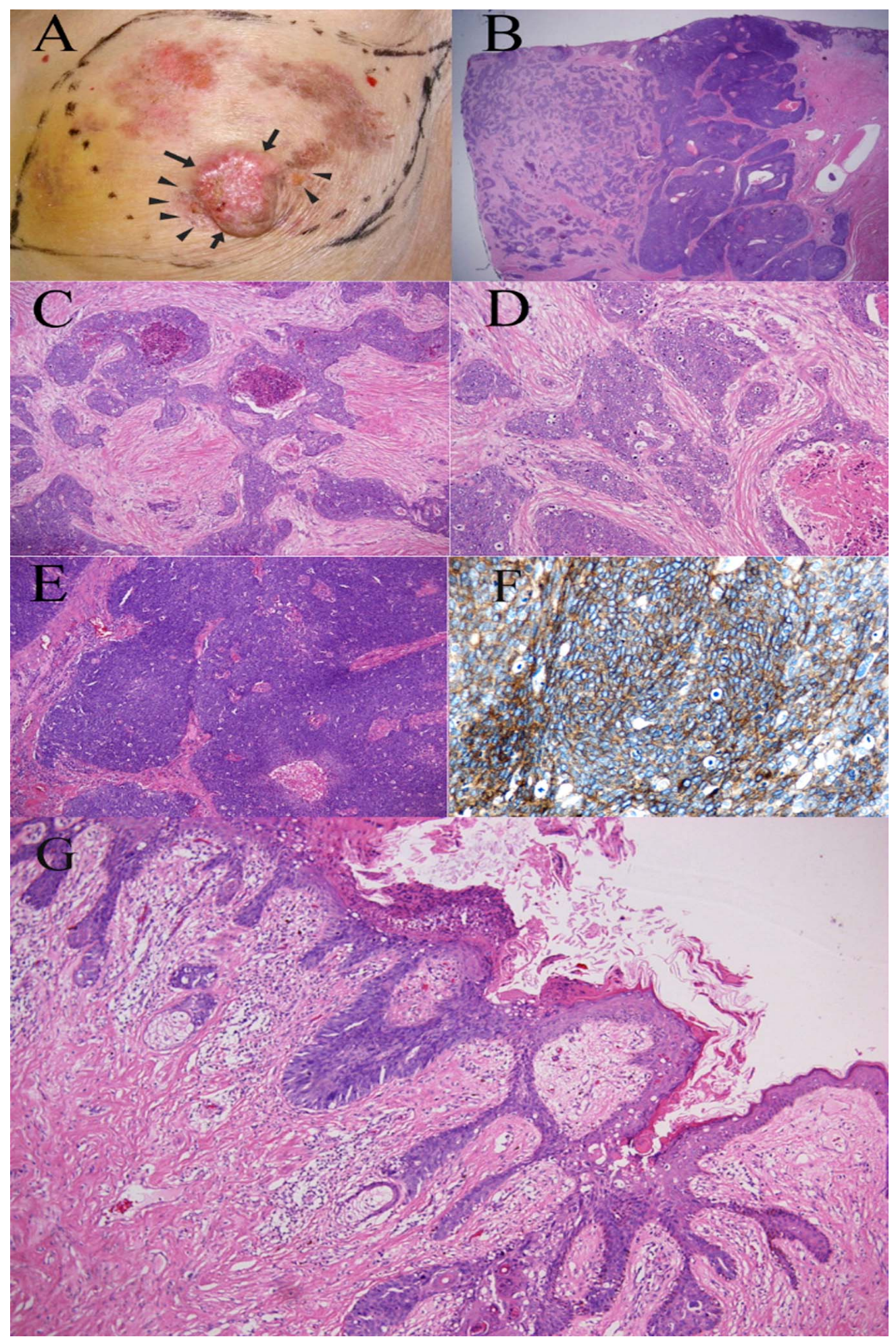

Fig. (1). Clinical appearance and histopathological findings of our case. (A) Ulcerated tumor with adjacent erythematous plaque (triangles) on the nipple of the left breast (arrows). Subcutaneous hemorrhage and erosions on the breast were the result of an accidental fall. (B) Multiple irregular-shaped tumor nests have invaded the entire dermis (x40, H\&E staining). (C) Massive necrosis was observed in some areas (x200, H\&E staining). (D) Tumor cells of various sizes and shapes, featuring individual keratinization and atypical mitotic figures (x200, H\&E staining). (E) The other comparatively larger masses consisted of hyperchromatic small squamous cells with basophilic cytoplasms (x200, H\&E staining). (F) Immunohistochemistry of EGF receptor (EGFR) in the tumor. The tumor cells were stained positively for antiEGFR antibody (EGFR pharmDx, K1492, DAKO) (x200). (G) Surgical specimen of the adjacent erythematous plaque (triangles in (A)) revealed irregular elongation of overlying epidermis, which contained atypical keratinocytes with pleomorphic nuclei, suggesting a diagnosis of Bowen's disease (SCC in situ) (x200, H\&E staining). 
Since these findings were not sufficient to identify the origin of this tumor, we carefully rechecked the histopathology of the surgical specimens and found irregular elongation of the overlying epidermis at the peripheral area corresponding to the adjacent erythematous plaques (Fig. $\mathbf{1 A}$, triangles), which contained atypical keratinocytes with pleomorphic nuclei, suggesting a diagnosis of Bowen's disease (SCC in situ) (Fig. 1G). We therefore concluded that tumor cells of Bowen's disease in the epidermis, independently from the tumors of liver and bile duct, had invaded downward, finally reaching the breast tissue. Although this invasion may have occurred partially through mammary ducts, no apparent infiltration into the ducts could be observed. In addition, there was no evidence of immunosuppression, a feature of some patients with Bowen's disease. After six months the patient was free from any local complications and was able to have a satisfactory QOL.

\section{COMMENTS}

The abdominal CT image did not show any intraabdominal lymph node metastases, suggesting that the tumors in liver and bile duct were unlikely to metastasize to the breast skin. Moreover, mammary cancers such as SCC of glandular epithelium and squamous metaplasia of ductal carcinoma were ruled out because immunohistochemical markers for these tumors, such as ER, PR, ErbB2/HER2, GCDFP-15 and CEA, were negative. On the other hand, a basal-like subtype of breast cancer, which is commonly triple-negative for ER, PR and ErbB2/HER2 [1], could not be ruled out from our immunohistochemical findings. Metastasis into the skin was suggested by the ulcerative appearance of the tumor but rechecking the histopathology of the surgical specimens resulted in detection of the overlying dysplastic epidermis (SCC in situ) (Fig. 1F) as the only clue [2] for identification of the origin of SCC in our case. Breast cancer or epidermotropic SCCs from other sites invading the epidermis usually show pagetoid features $[2,3]$ but such findings could not be observed in our case. In addition, epidermotropic SCC reportedly features smooth continuity to the epidermis, providing intercellular bridging between malignant tumor cells and benign squamous cells [4, 5]. However, while atypical keratinocytes with pleomorphic nuclei proliferated throughout the irregularly elongated epidermis (Fig. 1F), neither an actual connection of the tumor to the epidermis nor the pagetoid phenomenon was seen in our case. Further, this proliferation was observed at the periphery, but not at the center of the proliferating tumors beneath the epidermis. From these findings, continuity to the epidermis of an epidermotropic SCC from other origins could be ruled out.

\section{REFERENCES}

[1] Rakha EA, Reis-Filho JS, Ellis IO. Basal-like breast cancer: a critical review. J Clin Oncol 2008; 26: 2568-81.

[2] Brownstein MH, Helwig EB. Metastatic tumors of the skin. Cancer 1972; 29: 1298-307.

[3] Saruk M, Olsen TG, Lucky PA. Metastatic epidermotropic squamous cell carcinoma of the vagina. J Am Acad Dermatol 1984; 11:353-6.

[4] Weidner N, Foucar E. Epidermotropic metastatic squamous cell carcinoma. Report of two cases showing histologic continuity between epidermis and metastasis. Arch Dermatol 1985; 121: 1041-3.

[5] Youngberg GA, Berro J, Young M, Leicht SS. Metastatic epidermotropic squamous carcinoma histologically simulating primary carcinoma. Am J Dermatopathol 1989; 11: 457-65. 